\title{
Adaptation of Unified Dosimetry Index (UDI) and Critical Organ Scoring Index (COSI) for Target and Organ at Risk (OAR) in Prostate IMRT Technique
}

\author{
Memoona Kousar \\ Department of Radiation Oncology, Aga Khan University, Hospital \\ National stadium road, Aga khan university Karachi city Sindh 74800 \\ Abdul Qadir Jangdha \\ Department of Radiation Oncology, Aga Khan University, Hospital \\ National stadium road, Aga khan university Karachi city Sindh 74800
}

\begin{abstract}
The aim of the study is to formulate a dosimetry index that can be used for Quality assessment of IMRT treatment plans of prostate site. To compare different radiotherapy plans different indices are utilized. The present study will attempt to combine all the indices into one unified quality index which can define the quality of a treatment plan and serve as a quality indicator of treatment plan developed among similar and different modalities. This quality index will incorporate homogeneity index, conformity index, dose gradient and coverage index resulting in a direct comparison to an ideal plan. We developed another index in this study related to sparing of organs i.e. UDI (OARs) that incorporate the doses of OARs to compare which plan is optimum in terms of target coverage and OARs sparing. In comparison to this UDI (OARs) another work was done to analyze the dosimetry of overall plan including target coverage and organ at risk sparing i.e. critical organ scoring index (COSI). To validate UDI (Target), UDI (OARs) and COSI, 22 patients were studied which were planned on IMRT. We also define the ranking of the plan according to their target coverage and organ sparing quantities as 'Excellent', 'Good', 'Average' or 'Poor' plan. UDI index for the target and organ at risk is found to be a useful tool to quantify a treatment plan quality when volumes with different dose prescription are treated.
\end{abstract}

Keywords: Treatment planning system, Conformity index, Homogeneity index, Dose coverage, Dose gradient, Critical organs

DOI: $10.7176 / \mathrm{CMR} / 12-1-04$

Publication date: January $31^{\text {st }} 2020$

\section{Introduction}

Cancer is among the leading cause of deaths globally; including developing and developed countries. As $3^{\text {rd }}$ world countries around the world become more socially affluent, cancer deaths are expected to rise significantly due to combination of lifestyle changes and rapidly degradation of environmental factors [1]. According World Health Organization(WHO), developing countries account for nearly $72 \%$ of cancer death even though the average disease occurrence in these countries is lower in comparison to that of first world nations [1]. It is estimated that by 2030 , the number of new cancer cases will mount to 21.7 million and 13 million patients will die owing to the growth and aging of the population. Prostate cancer is the third most common cancer in men and is expected to increase internationally [2]. Intensity modulated radiation therapy (IMRT) has been shown to be the preferred delivery method for prostate cancer [9]. For the evaluation of any treatment plan some tools are necessary to judge how accurate the plan meets the optimum objectives. The purpose of the treatment planning is to provide full dose to the target volume so it could have high conformity and homogeneity and to reduce the risk factors of organ at risks and limiting the doses of organ at risk below their tolerance limit.

For the evaluation of treatment plan several indices could be used in separate as well as combined scoring system. Several indices are suggested for the evaluation of treatment plan which include homogeneity index conformity index dose coverage and dose gradient. Akpati et al. introduced the scoring system named UDI which incorporate all the indices mentioned above for the evaluation of target coverage [5]. Following UDI implementation this work developed the scoring system for organ at risk i.e. UDI (OAR) and Critical Organ Scoring Index (COSI) as well for the specific prostate IMRT.

\section{Materials and Methods}

\subsection{Development of UDI for Target coverage}

Conformity index is defined as an extension of section-by-section dosimetric analysis and dose-volume histograms and can be defined as an absolute value resulting from the ratio between a fraction of the tumor volume and the volume covered with the certain isodose line [10]. Dose Homogeneity is defined as the ratio of the difference between the near-maximum and near-minimum absorbed dose to the median dose. Dose coverage index measures 
how well the PTV is covered by the prescribed dose. It is defined as the ratio of PTV receiving the Prescribed dose. Whereas dose gradient Index describes the decrement of dose in high dose region and used to describe the relationship between the volume of tumor covered by the prescribed dose and the volume covered by the half of the prescribe dose. The ideal value of these indices defined above i.e. dose conformity, dose gradient and dose coverage is equal to 1 and dose homogeneity to score 0.0 for an ideal plan.

Indices formulations, used in this study, are the following

$$
\begin{aligned}
& \text { Conformity index }=\frac{T V_{R I}}{T V} X \frac{T V_{R I}}{V_{R I}}, \\
& \text { Homogeneity Index }=\frac{D 2 \%-D 98 \%}{D 50 \%} \\
& \text { Coverage Index }=\frac{T V_{R I}}{T V} \\
& \text { Gradient Index }=\frac{V_{R I}}{V 50 \%}
\end{aligned}
$$

Where $\mathrm{TV}_{\mathrm{RI}}$ is the PTV receiving the $95 \%$ of the prescribed dose, TV is the target volume of prostate, $\mathrm{V}_{\mathrm{RI}}$ is the volume receiving $95 \%$ of the prescribed dose, $\mathrm{D}_{2} \%$ is the volume receiving $2 \%$ of the prescribed dose (nearmaximum dose) and D98\% is the volume receiving 98\% of the prescribed dose (near-maximum dose) and D50\% is the volume receiving $50 \%$ of the prescribed dose and V50\% is the volume receiving $50 \%$ of the prescribed dose. The UDI (Target) formula based on mathematical logic is defined as;

$$
\mathrm{UDI}=\left(\sum_{n=4}^{1} w k\left(\left|1.0-D I_{K}\right|+0.1\right) \times 10^{4}\right.
$$

Where $\mathrm{DI}_{\mathrm{K}}$ are the indices i.e. dose coverage, dose conformity, dose homogeneity and dose gradient. $\mathrm{W}_{\mathrm{k}}$ denotes the weighting factor that explain the overall importance of individual index. To prevent inflation of the UDI score the weightage factor should be equal to 1 , i.e. W1 $\mathrm{x} \mathrm{W} 2 \times \mathrm{W} 3 \times \mathrm{W} 4=1$ [5]. To analyze the individual index of UDI the indices whose ideal value is equal to 1.0 can be expressed in some generic function UDI (X) which can be defined as;

Where $\mathrm{X}=\{\mathrm{CI}, \mathrm{DG}$ and $\mathrm{C}\}$

$$
\mathrm{UDI}(\mathrm{X})=\{|1.0-\mathrm{X}|+0.1\} \times 10
$$

\subsubsection{For the homogeneity index}

$$
\mathrm{UDI}(\mathrm{HI})=\{|\mathrm{HI}|+0.1\} \times 10
$$

\subsection{Development of UDI for OARs}

Another scoring index is developed for OARs i.e. UDI (OAR) to evaluate the best optimum plan in term of Organ at Risk. For this purpose, QUANTEC protocol for ca prostate were followed in order to analyze the organs with their respective tolerance doses as mentioned in the protocol (Table 1).

$$
\mathrm{UDI}_{(\mathrm{OARs})}=\prod_{n=4}^{1} w k\left\{\sum_{n=4}^{1} \frac{\operatorname{VOARS}(\text { Planning })}{\operatorname{VOARS}(\text { Protocol })}\right\}
$$

Where $\mathrm{V}_{\text {OARs }}$ (Planning) is the volume of OARs extracted from the DVH of dosimetric plan and $\mathrm{V}_{\text {OARs }}($ protocol) is the standard volume as per QUANTEC protocol. $\mathrm{W}_{\mathrm{k}}$ denotes the weighting factor that explain the overall importance of individual organ). Different weightages were assigned to each organ with respect to their late toxicity effects and clinical importance. For example, Rectum is the most important organ in clinical aspect so greater value of weightage were assign to this organ as shown in Table 2

\subsection{Development of Critical Organ Scoring Index}

Another work was done simultaneously for the scoring of OARs to analyze the optimum plan in terms of OARs sparing and target coverage i.e. Critical Organ Scoring Index (COSI). COSI is a measure of both, i.e. doses to target and critical organ at risk. To accomplish this, Histogram Analysis Radiotherapy (HART) software was followed to extract the formula which is used for the study. All calculation was done in Microsoft Excel. It can be expressed as:

$$
\operatorname{COSI}=\left[1-\frac{\sum_{i=1}^{N} V(O A R S)}{T C I}\right]
$$

Where $\mathrm{V}_{(\mathrm{OARs})}$ is the fractional volume of $\mathrm{i}^{\text {th }}$ organ at risk (OAR) receiving more or less than tolerance dose and $(\mathrm{Wi})$ is the relative weight of fractional volume of each organ, and TCI is the total coverage index of the treatment plan. For an ideal dosimetry of plan, this index should be equal to $=1.0$

\section{Results}

To evaluate the UDI scoring index for both target coverage and OARs, 22 patients of prostate cancer, planned with 
IMRT Technique were randomly selected over a period of 2 years.

For the evaluation of UDI(Target) and UDI (OAR), the mean and standard deviation were calculated for all dosimetry indices and for UDI as well. UDI values were collected and plotted to quantitatively compare and classify the results. Four classification groups were established based on the mean and standard deviation such that UDI scores greater than $(\mu+\sigma)$ were classified as poor, whereas scores from $u$ to $(\mu+\sigma)$ classified as average, UDI scores from $\mu$ to $(\mu-\sigma)$ were classified good and score less than $((\mu-\sigma)$ were excellent. Out of 55 treatment plans (20 patients), only $9(5.85 \%)$ plans resulted "poor" plans and $11(7.15 \%)$ plans were resulted as "excellent" plans. The most of the treatment plans were classified as "average" and "good" plans. The lowest value of UDI (Target) is 23, whereas the highest value of UDI (Target) is 63.97. Similar work was done for the evaluation of UDI (OAR) where, out of 20 cases, the minimum deviation of UDI is 0.20 and maximum deviation is 0.44 . as shown in fig 1 and 2. Figure 3 below show the UDI of target which explain the variation of different plans. The lowest score which represent the minimum deviation from an ideal plan (i.e. $=1$ ) is 23 whereas the highest score represents the maximum deviation from an ideal plan is 63.91. The overall evaluation of each indices for an individual plan which include dose coverage (DCI), dose conformity (CI), dose homogeneity (HI) and dose gradient (DG) is shown in figure 4. Dose gradient is the most influential component for UDI b/c of relatively high magnitude of deviation from reference (ideal plan) values.

\section{DISCUSSION}

The dosimetry scoring method is a beneficial tool for the evaluation of quantitative and qualitative plan. It can also be used as a comparison tool for different plans of different modality

\subsection{UDI (OAR) comparison with UDI (Target)}

After evaluating all the data comparison was done between the UDI (OAR) and UDI (Target) just to observe the consistency and comparative result. It was observed that the case with excellent UDI (Target) score has the best UDI (OAR) value which means that UDI (Target) and UDI (OAR) are comparative to each other. Case with the worse target coverage has the worse sparing of OARs and case with good target coverage has the good sparing of OARs. As mentioned in table 2.

To demonstrate the effect of UDI (Target) scoring method on dose distribution of worse to good plan, UDI score is associated, worse UDI score has worse dose distribution and good UDI score has good dose distribution as shown in figure 5.

The dose distributions in case 5 show better dose conformity and dose homogeneity compared to the dose distribution in case 9 which is consistent with UDI score.

Corresponding dose volume histogram (DVH) plots for both cases from worse to good for evaluating the dose coverage and organ at risk dose distribution. Evaluation of the DVH shows that both cases receive good dose coverage of PTV meeting the standard protocol i.e. (95\% of the volume should receive $95 \%$ of the dose). But if we evaluate the doses of OARs (i.e. Rectum and bladder) it can be seen that in case 5 there is better dose distribution of OARs as compared to case 9, as shown in Figure 6

\subsection{UDI (Target) and UDI (OAR) Comparison with COSI}

Out of 55 cases, case 9 has worse UDI score which is 63.90 and case 5 has good UDI score which is 23.0 in term of target and OAR as well, both UDI (Target) and UDI (OAR) further compare with the COSI scoring system just to analyze the variation between each scoring system and comparative results, the worse value of COSI represent the worse UDI (OAR) and UDI (Target) and good value of COSI value represent the good value of UDI(OAR) and UDI (Target) as shown in Table 3.

\section{CONCLUSION}

A scoring system developed for the evaluation of a dosimetry plan for IMRT prostate cancer. The proposed plan quality scoring system unifies four indices for the evaluation of target coverage into one simple equation which translate the overall quality of individual dosimetry plan. This work also proposed a similar method for creating a scoring system for organ at risk in which all the ratios of organs are covered into one equation that can be easily utilized for calculating a figure of merits and quantifies the overall quality of optimum dosimetry plan. UDI (OARs) and UDI (Target) were compared with Critical structure scoring index (COSI) and found to be in good agreement to validate the scoring of dosimetry of treatment plan. UDI index for the target and organ at risk is found to be a useful tool to quantify a treatment plan quality when volumes with different dose prescription are treated. Under this scoring system, for a collection of treatment plans, the quality of plans can be classified as 'Excellent' 'Average' 'Good' and 'Poor' in term of target coverage and OARs. This proposed indexing system is not limited to only IMRT plans, but can be extended to compare the quality of plans that may be produced by other treatment techniques, like 3DCRT, IMRT, VMAT, SRS, etc. The proposed system can also be utilized to evaluate the plan quality of any other treatment sites by adjusting the OAR tolerance criteria following the clinical significance and 
standard protocols.

\section{References}

[1] V. Sharma, S. H. Kerr, Z. Kawar, and D. J. Kerr, "Challenges of cancer control in developing countries: Current status and future perspective," Futur. Oncol., vol. 7, no. 10, pp. 1213-1222, 2011.

[2] G. T. Borges et al., "Conversion of Prostate Adenocarcinoma to Small Cell Carcinoma-Like by Reprogramming," J. Cell. Physiol., vol. 231, no. 9, pp. 2040-2047, 2016.

[3] J. E. Damber, "Prostate cancer: Epidemiology and risk factors," Curr. Opin. Urol., vol. 8, no. 5, pp. 375$380,1998$.

[4] T. Hira, "Dosimetric Comparison between the Intensity Modulated Radiation Therapy and Volumetric Modulated arc Therapy for Prostate Cancer- A Case Study," vol. 2, no. 1, pp. 1-3, 2014.

[5] H. Akpati, C. S. Kim, B. Kim, T. Park, and A. Meek, "Unified dosimetry index (UDI): A figure of merit for ranking treatment plans," J. Appl. Clin. Med. Phys., vol. 9, no. 3, pp. 99-108, 2008.

[6] E. E. Nelson and A. E. Guyer, "NIH Public Access," vol. 1, no. 3, pp. 233-245, 2012.

[7] C. Ceylan, N. Kucuk, H. Bas Ayata, M. Guden, and K. Engin, "Dosimetric and physical comparison of IMRT and CyberKnife plans in the treatment of localized prostate cancer," Reports Pract. Oncol. Radiother., vol. 15, no. 6, pp. 181-189, 2010.

[8] M. Mambretti et al., "A global Unified Dosimetry Index (gUDI) to evaluate simultaneous integrated boost radiotherapy plans in prostate cancer," Radiother. Oncol., vol. 128, no. 2, pp. 315-320, 2018.

[9] V. Kannan, S. Deshpande, V. Anand, Y. Ghadi, and S. De, "High-dose intensity-modulated radiotherapy as primary therapy for prostate cancer: Report on dosimetry aspects and acute toxicity in the Indian scenario," J. Cancer Res. Ther., vol. 6, no. 1, p. 58, 2010.

[10] I. Paddick, "A simple scoring ratio to index the conformity of radiosurgical treatment plans. Technical note.," J. Neurosurg., vol. 93 Suppl 3, no. Suppl 3, pp. 219-22, 2000.

Table 1: OARs Doses from QUANTEC protocol followed for the study.

\begin{tabular}{|l|l|c|}
\hline Organ & End Point & Dose Volume Parameter \\
\hline Rectum & Grade $\geq 2 / \geq 3$ late toxicity & V $50<50 \%$ \\
& Grade $\geq 2 / \geq 3$ late toxicity & $\mathrm{V} 60<35 \%$ \\
& Grade $\geq 2 / \geq 3$ late toxicity & $\mathrm{V} 65<25 \%$ \\
& Grade $\geq 2 / \geq 3$ late toxicity & $\mathrm{V} 70<20$ \\
& Grade $\geq 2 / \geq 3$ late toxicity & $\mathrm{V} 75<15 \%$ \\
\hline Bladder & Grade $\geq 3$ late toxicity & $\mathrm{V} 65 \leq 50 \%$ \\
& & $\mathrm{~V} 70 \leq 35 \%$ \\
& & $\mathrm{~V} 75 \leq 25 \%$ \\
\hline Bowel Bag & & Mean $<35$ \\
\hline Femoral Heads & Severe erectile dysfunction & Mean $<5$ \\
\hline
\end{tabular}

Table 2: Organ at risk weightages with respect to their late toxicity effects and clinical importance.

\begin{tabular}{|c|c|}
\hline Organ at Risk & Weightage \\
\hline Rectum & 0.4 \\
\hline Bladder & 0.25 \\
\hline Bowel Bag & 0.05 \\
\hline Femoral Heads & 0.15 \\
\hline
\end{tabular}

Table 3: Illustration of UDI comparison between UDI (OARs) and UDI(T). Table explain the UDI target with worse score has the worse value of organ at risk sparing and the score with the minimum deviation of target coverage has the best score of Organ at Risk

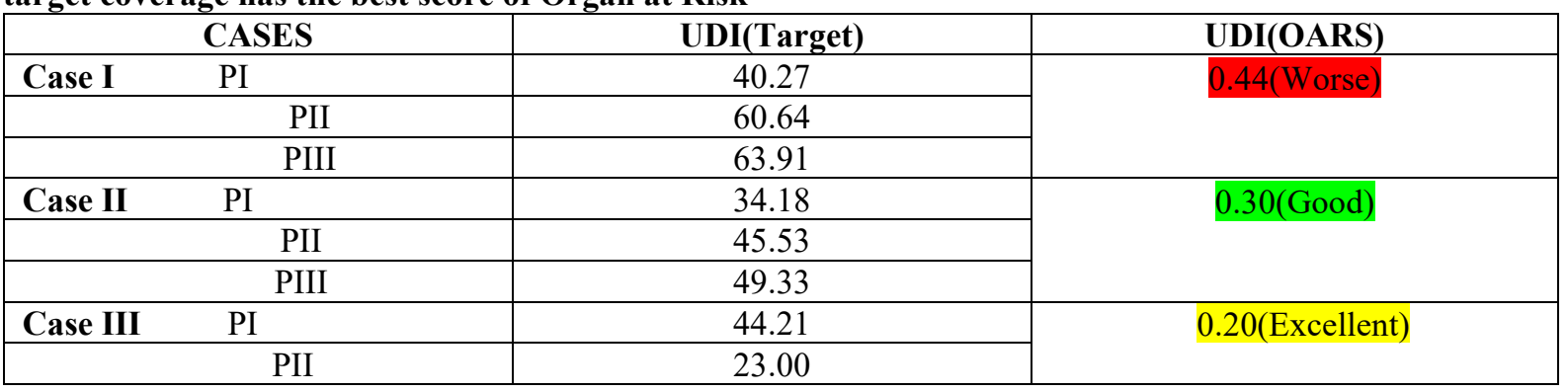


Table 4: Illustration of scoring system comparison between UDI (OARs), UDI (Target) and COSI. Table explain the UDI target with worse score has the worse value of organ at risk sparing and COSI and the score with the minimum deviation of target coverage has the best score of Organ at Risk and COSI.

\begin{tabular}{|c|c|c|c|}
\hline CASES & UDI (Target) & UDI (OARS) & COSI \\
\hline CaseI & 40.27 & \multirow[t]{3}{*}{ 0.44(Worse) } & \multirow[t]{3}{*}{-5.58 (Worse) } \\
\hline PII & 60.64 & & \\
\hline PIII & 63.91 & & \\
\hline CaseII & 34.18 & \multirow[t]{3}{*}{0.30 (Good) } & \multirow[t]{3}{*}{$-2.94($ Good $)$} \\
\hline PII & 45.53 & & \\
\hline PIII & 49.33 & & \\
\hline Case III & 44.21 & \multirow[t]{2}{*}{0.20 (Excellent) } & \multirow[t]{2}{*}{-1.56 (Excellent) } \\
\hline PII & 23.00 & & \\
\hline
\end{tabular}

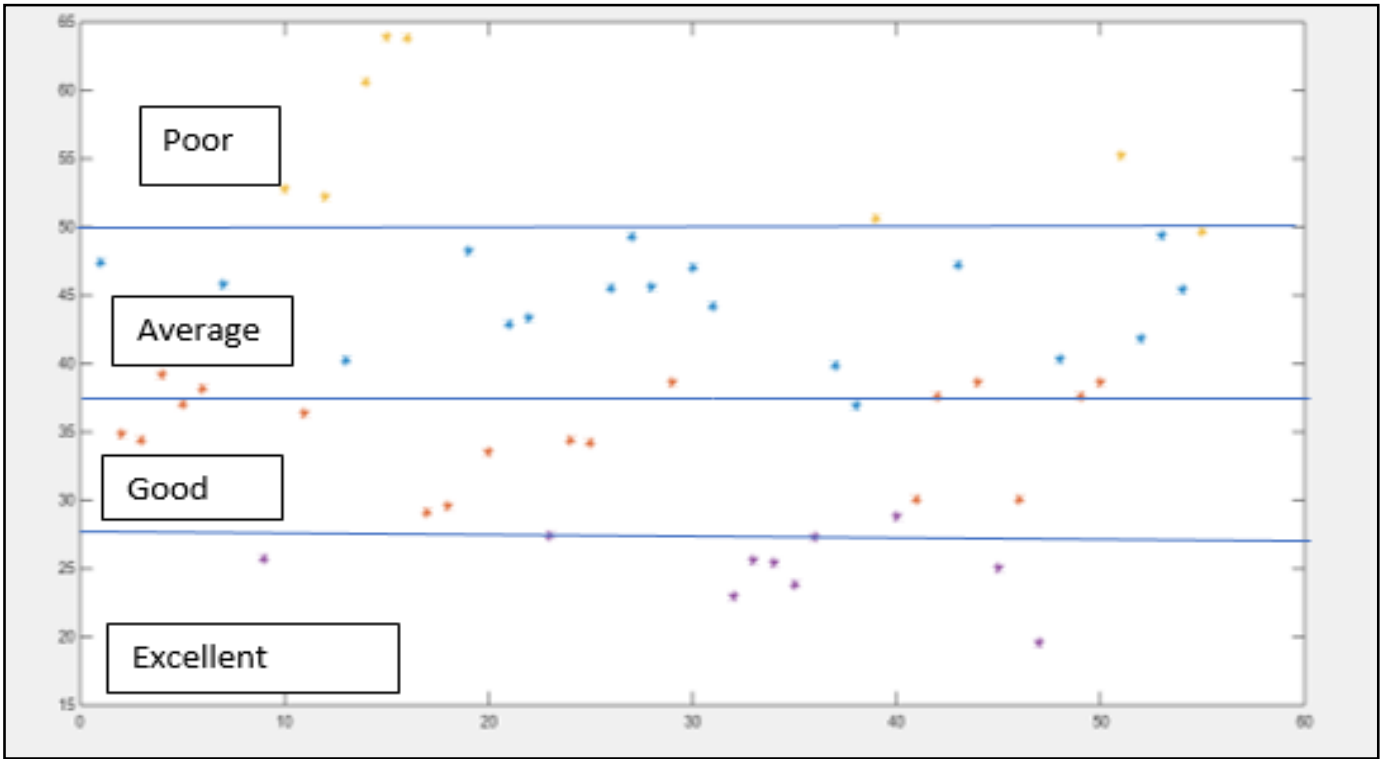

Figure 2:UDI (Target) ranking system based on mean and standard deviation. Ranked as 'Excellent' 'good' 'average' and 'poor'

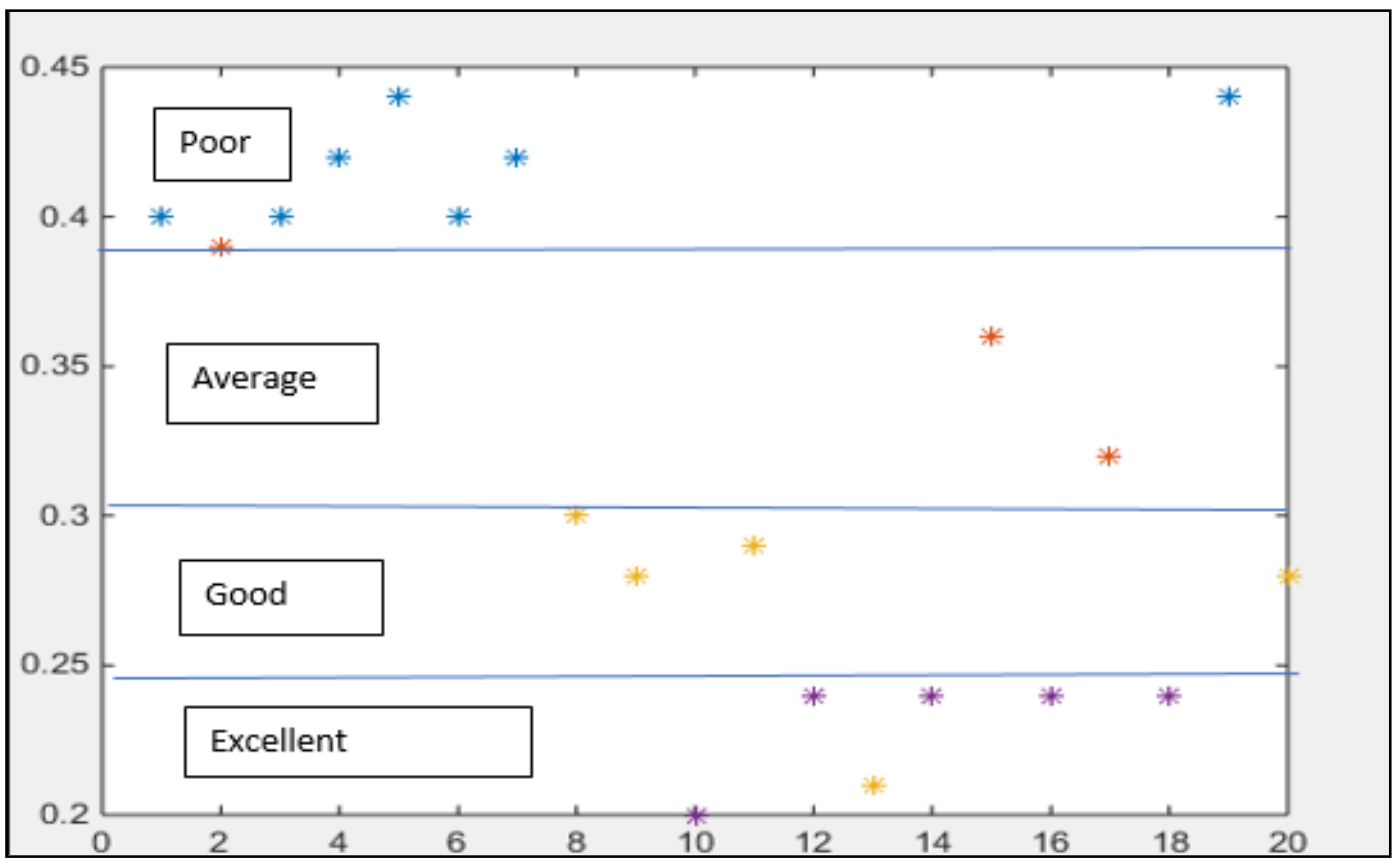

Figure 3: UDI for organ at risk ranking system based on mean and standard deviation obtained for 20 cases 


\section{UDI (Target)}

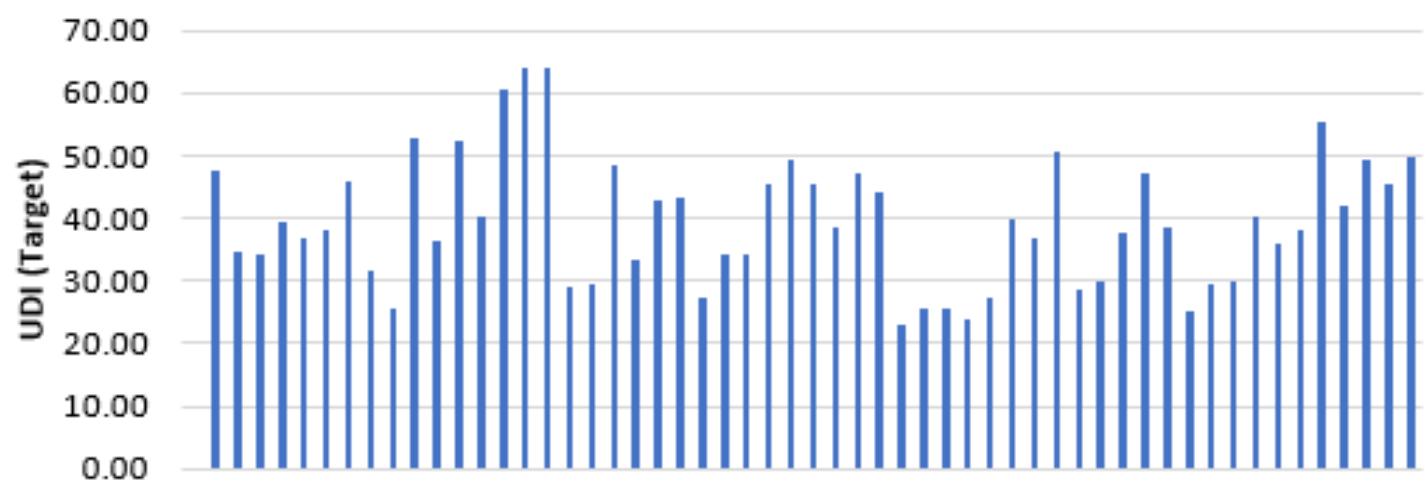

$1 \quad 3 \quad 5 \quad 7 \quad 91113151719212325272931333537394143454749515355$

Treatment Plans

Figure 4: histogram plot of unified dosimetry index for 55 IMRT treatment plans

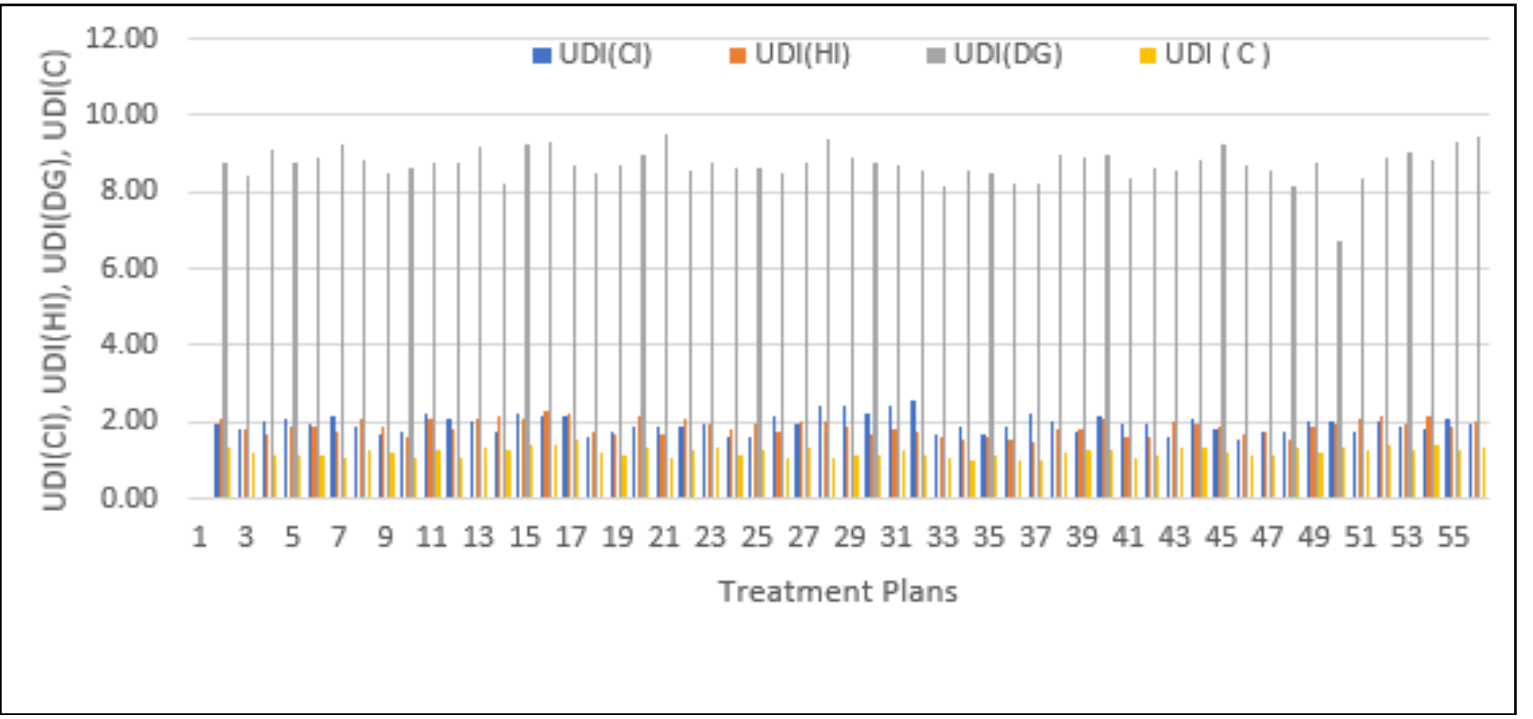

Figure 5: Histogram of UDI that contribute all four individual indices in term of equation, UDI(C), UDI(CI), UDI(HI), UDI(DG) which denotes coverage, dose homogeneity, dose gradient and, conformity index 

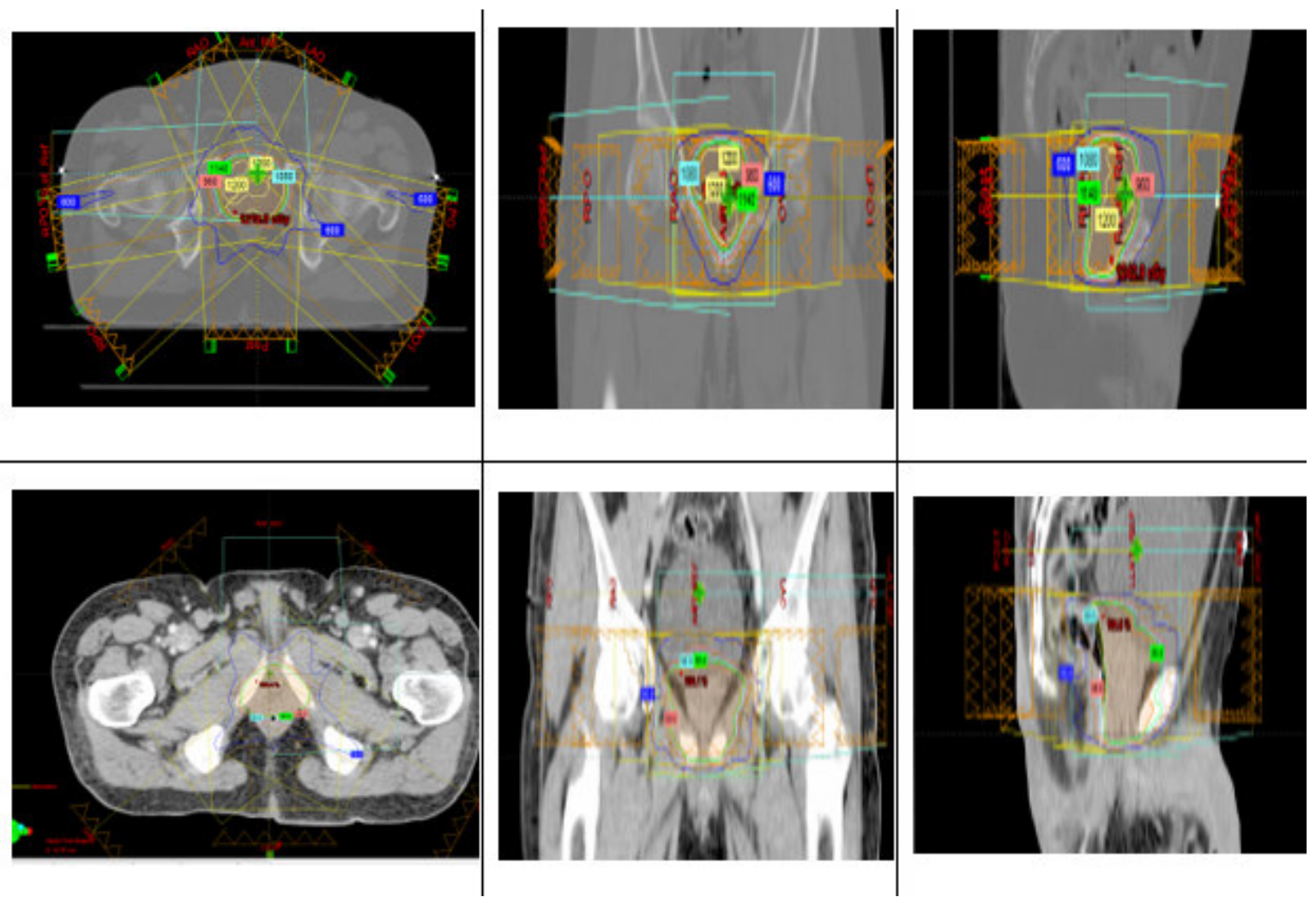

Figure 6: Dose distribution of worse and good UDI score. Plot showing axial, coronal and sagittal view of dose distribution of cases with good UDI on top and worse UDI on bottom. The corresponding UDI score were 63.91 and 23.0 , respectively
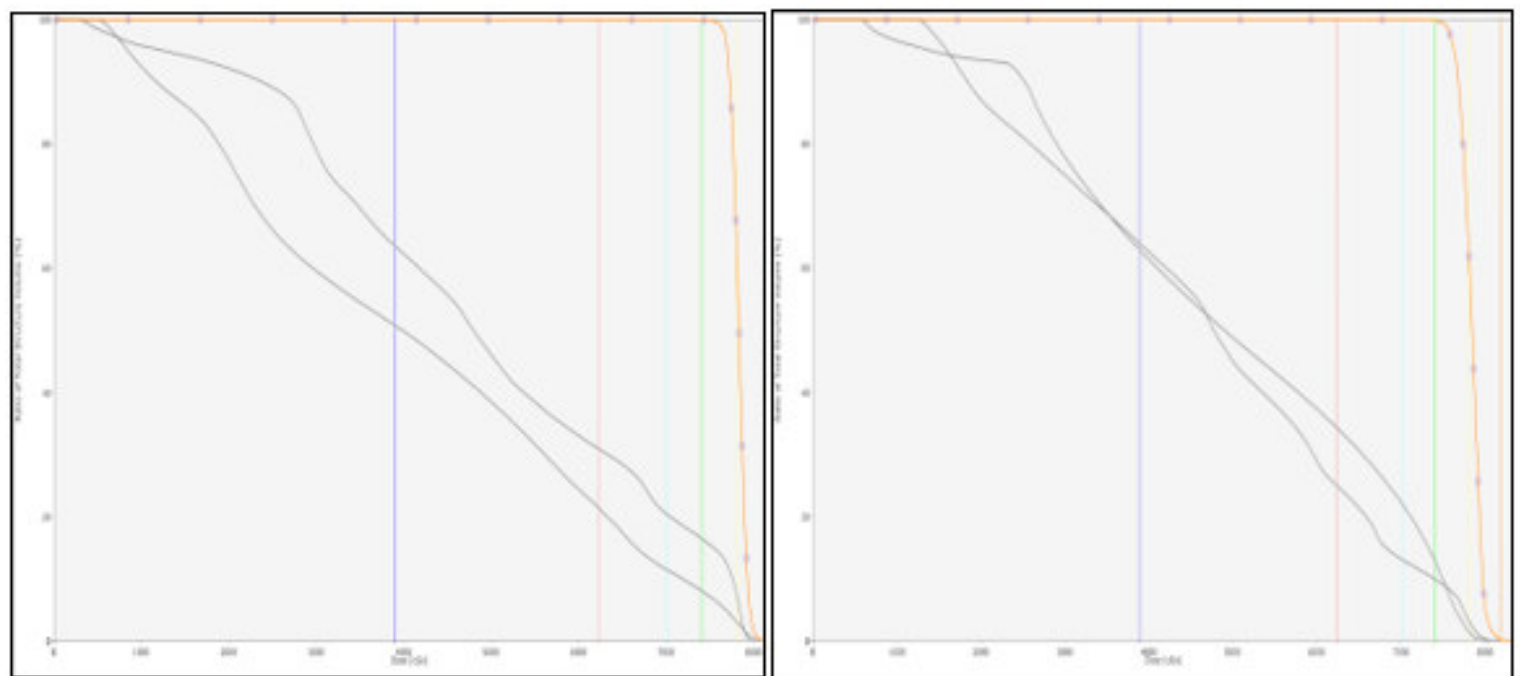

Figure 7:Dose Volume Histogram (DVH) plots for PTV and OARs for case 5 on Left and case 9 on Right 